\title{
Triage of patients for short term observation after elective coronary angioplasty
}

\author{
K T Koch, J J Piek, M H Prins, R J de Winter, K Mulder, K I Lie, J G P Tijssen
}

\begin{abstract}
Objective-To evaluate triage of patients for short term observation after elective percutaneous transluminal coronary angioplasty (PTCA), as appropriate selection of patients for short term observation after angioplasty may facilitate early discharge.

Methods-1015 consecutive patients scheduled for elective PTCA were prospectively included for short term observation. Patients with unstable angina Braunwald class III were excluded. There were no angiographic exclusion criteria. Patients were discharged from the interventional centre when considered stable during 4 hours of observation after PTCA. It was left to the operator's discretion whether to prolong the observation period. Procedural complications were defined as death, coronary bypass surgery, early repeat PTCA, and myocardial infarction.

Outcome measures-The need for prolonged observation ( $>4$ hours) and the occurrence of complications. Predictors for prolonged observation and the occurrence of complications after the 4 hours observation were assessed by univariate and multivariate analysis.

Results-Two patients died, including one of six patients who underwent emergency bypass surgery. In all, 922 patients $(90.8 \%)$ were triaged to short term observation and had an uncomplicated three day follow up. Observation was prolonged in 87 patients $(8.6 \%)$, and 40 patients had a complicated course. Independent predictors of procedural complications were acute closure (odds ratio (OR) 9.7; 95\% confidence interval 4.4 to 21.4 ), side branch occlusion (OR 8.9; 3.4 to 23.7), no angiographic success (OR 5.1; 2.4 to 11.0 ), female sex (OR 3.1, 1.7 to 5.7), any unplanned stent (OR 2.8, 1.4 to 5.9), and ostial lesion (OR 2.2, 1.0 to 4.7).

Conclusions-A 4 hour observation period is safe after elective coronary angioplasty. As procedural variables are the strongest predictors of postprocedural complications, the immediate procedural results allow effective triage of patients for short term or prolonged observation in order to anticipate complications.
\end{abstract}

(Heart 2000;83:557-563)

Keywords: angioplasty; triage; interventional cardiology

The wide application of percutaneous transluminal coronary angioplasty (PTCA) as a method for myocardial revascularisation has led to increasing logistic constraints. ${ }^{1}$ Reduction of hospital stay and performance of outpatient procedures are recommended by health care insurers and hospital management in order to reduce costs. Furthermore, early discharge after PTCA may add to the comfort of the patients but may be complicated by unattended subacute occlusion of the treated vessel away from monitoring and angioplasty facilities.

Abrupt vessel closure and myocardial infarction are the major acute complications of coronary angioplasty. ${ }^{2-4}$ The risk of acute or subacute closure has been related to many clinical and angiographic features. ${ }^{-11}$ Taking these risk factors into account, only a limited number of patients would be eligible for short term observation after coronary angioplasty. On the other hand, it has been suggested that subacute closure remains largely unforeseeable and occurs out of laboratory in only a minority of patients. ${ }^{9}$ Furthermore, this complication is rare after a satisfactory angiographic result following balloon angioplasty or after stent implantation, whether this was done for suboptimal angioplasty or as a "bail out" device. ${ }^{9}{ }^{11-14}$
Our purpose in this study was to identify factors associated with the need to prolong the observation period after elective coronary angioplasty and stent implantation. We also evaluated prospectively the appropriateness of triage for either short term or prolonged observation.

\section{Methods}

The study group consisted of 1015 consecutive patients who underwent elective coronary angioplasty at the Academic Medical Centre in Amsterdam between January 1995 and May 1997. Inclusion for short term observation was based on referral: all patients referred to our centre for elective PTCA from hospitals within a range of 40 miles $(65 \mathrm{~km})$ were included and studied prospectively. There were no angiographic exclusion criteria. Patients with multivessel disease, previous PTCA or coronary bypass surgery, or impairment of left ventricular function were not excluded. Exclusion criteria were scheduled angioplasty with intraaortic balloon pump assistance or under general anaesthesia, and angioplasty performed for acute myocardial infarction or for unstable angina classified as Braunwald class III. ${ }^{15}$ Patients with unstable angina classified as Braunwald class I and II were not excluded. 
CLINICAL AND ANGIOGRAPHIC DATA COLLECTION Preprocedural clinical characteristics were collected prospectively, including risk factors for coronary artery disease, previous myocardial infarction, previous coronary angioplasty or bypass surgery, and anginal symptoms classified according to Canadian Cardiovascular Society and Braunwald criteria. Preprocedural angiographic data were collected prospectively and included the number of diseased vessels, lesion location according to the coronary artery surgery study, ${ }^{16}$ and lesion severity graded visually according to an ordinal scale ( $<50 \%$, $50-75 \%, 75-90 \%, 90-99 \%$, totally occluded).

Single vessel disease was defined as one diseased vessel - that is, at least one lesion with $>50 \%$ reduction in lumen diameter; multivessel disease was defined as two or more diseased vessels. Coronary flow was graded according to TIMI criteria. ${ }^{17}$

The morphological characteristics of the lesion were assessed using the following definitions:

Eccentricity: concentric or eccentric (asymmetrically positioned in one or more views).

Length: estimate of length of the stenosis using the guiding catheter for calibration $(<5$, 5 to 10,10 to $20,>20 \mathrm{~mm}$ ).

Angulation: bending of the lesion, graded as mild or absent $\left(<45^{\circ}\right)$, moderate $\left(45^{\circ}-60^{\circ}\right)$, or excessive $\left(>60^{\circ}\right)$.

Calcification: radiodensities present or absent within the vessel wall noted angiographically at the site of the lesion.

Thrombus: intraluminal filling defect noted at angiography.

Ostial: involvement of the origin of the right, left anterior descending, or left circumflex coronary artery.

Side branch involvement: involvement or not of side branch vessels with diameter $>2 \mathrm{~mm}$.

Bifurcation lesion: a lesion involving two major arterial branches, the smaller being $>2 \mathrm{~mm}$ in diameter or considered suitable for bypass surgery. The side branch had a $>50 \%$ diameter stenosis at its origin.

Protected side branch: angioplasty guidewires inserted in both the main and side branches.

Unprotected side branch: guidewire could not be inserted or was not inserted in either of the coronary branches.

Coronary occlusion: defined as functional in the presence of impaired antegrade flow (TIMI-1), and as total in the absence of antegrade flow (TIMI-0).

The duration of the occlusion was determined from previous angiographic information or estimated using an index clinical event such as a myocardial infarct or a sudden change in anginal symptoms. Coronary lesions were also classified using morphological characteristics according to the American College of Cardiology/American Heart Association criteria ${ }^{18}$ and the modified lesion classification as suggested by Ellis. ${ }^{6}$

\section{ANGIOPLASTY PROCEDURE}

Elective angioplasty was performed by the femoral approach using $6 \mathrm{~F}$ guiding catheters routinely, except for atherectomy or the sched- uled use of $6 \mathrm{~F}$ incompatible stents. All patients were pretreated with $100 \mathrm{mg}$ of aspirin taken orally. A standard dose of 5000 IU of heparin was given as an intra-arterial bolus after insertion of the arterial sheath. Angioplasty was performed by experienced operators with standard interventional guiding catheters, guidewires, balloons, and stents, with on site surgical stand by. A low osmolar, ionic contrast agent (Hexabrix) was used. After stent implantation, patients were also treated with ticlopidine $250 \mathrm{mg}$ daily for one month.

The procedure was considered to have been completed if the angiographic result remained satisfactory after a five minute wait. The sheaths were removed immediately after completion of the procedure. Haemostasis was achieved by manual compression. A 12 lead ECG was obtained before and after completion of the procedure. The patients were observed in our centre for another four hours without telemetry. They were returned to their referring hospitals in a 40 mile radius to a normal bed without telemetry if they were free of symptoms and if a repeat ECG remained unchanged.

Patients were selected for prolonged observation on the following criteria: severe dissection with failed or suboptimal stent implantation, occluded side branch, angiographic evidence of thrombus, vessel perforation by the guidewire, or suspected cerebrovascular accident. It was left to the discretion of the attending interventional cardiologist at any time after the procedure to retain the patient for prolonged observation and monitoring in the interventional clinic, whether or not additional heparin treatment was given.

PROCEDURAL DATA COLLECTION

Successful angioplasty was defined as less than $50 \%$ residual stenosis and TIMI-3 flow, and procedural success as successful angioplasty without death from any cause, acute myocardial infarction, urgent coronary bypass surgery for failure of angioplasty, or repeat angioplasty within 48 hours. Single lesion angioplasty was defined as treatment of one coronary lesion, multilesion angioplasty as treatment of more than one lesion in the same vessel, and multivessel angioplasty as treatment of more than one vessel.

Data were collected for each lesion on the incidence of acute/temporary closure, side branch occlusion, spasm, "no reflow," and the angiographic appearance of thrombus. Dissections were noted as local or extensive and classified according to the National Heart, Lung, and Blood Institute classification system. ${ }^{19}$ The indications for stent implantation were classified by the operator as elective, bail out, or stent implantation for an unsatisfactory angiographic result.

Prolonged observation after angioplasty was defined as any prolongation of stay at our clinic longer than four hours after the procedure, whether or not with prolonged heparin treatment. The interventional cardiologist's reasons for prolonged observation and monitoring were documented. Where applicable, the lesion 
Table 1 Non-eligible patients

\begin{tabular}{lrr}
\hline & $n$ & $\%$ \\
\hline All procedures during study period & 2131 & \\
Not included because of: & & \\
$\quad$ Unstable angina Braunwald class III & 635 & 29.8 \\
Acute myocardial infarction & 245 & 11.5 \\
Intra-aortic balloon pump support & 22 & 1.0 \\
General anaesthesia & 8 & 0.4 \\
$\quad$ Miscellaneous & 206 & 9.7 \\
Included & 1015 & 47.6 \\
\hline
\end{tabular}

for which prolonged observation was considered necessary was indicated, in case the procedure involved angioplasty of more than one lesion. Procedural complications and clinical events during prolonged observation were documented. Clinical follow up data were obtained until discharge from the referring hospitals. Complications were defined as death from any cause, coronary bypass surgery or repeat PTCA during the hospital admission, myocardial infarction, or other complications requiring a hospital stay, such as pericardial drainage for perforation.

DATA ANALYSIS

All patients were included in the follow up analysis except those who died within four hours of the procedure or those undergoing emergency bypass surgery. Univariate logistic regression analysis was used to identify clinical and preprocedural morphological predictors of the need for prolonged observation after angioplasty, as well as to identify clinical, preprocedural, and procedural predictors of complications. Predictors with $\mathrm{p}<0.20$ using univariate logistic analysis were entered into the stepwise multivariate logistic regression analysis to identify independent predictors of the need for prolonged observation and the occurrence of complications. A logistic regression model was developed to predict complications after four hours of observation. The corresponding risk function was simplified by assigning points to each of the predictive variables. (The weights were assigned in proportion to the logarithm of the corresponding odds ratio.) The total risk score for each individual patient was calculated by adding the risk points that applied to that patient. Categorical data are presented as prevalence rates and continuous data are presented as mean (SD). All statistical analyses were performed using SAS software package (version 6.10 ). A $p$ value $<0.05$ was considered significant.

\section{Results}

During the study period, 2131 patients underwent a PTCA procedure at our centre. Table 1 summarises the reasons for exclusion of patients during the study period. Miscellaneous reasons for exclusion were, for example, admission to the clinical ward of our department or participation in study protocols necessitating in-hospital observation. The study population consisted of 1015 patients, involving a total of 1510 coronary lesions. The clinical and angiographic characteristics of the patients are summarised in table 2 .
The overall procedural success rate was $91.2 \%$. Four patients died during admission at the hospital $(0.4 \%, 95 \%$ confidence interval (CI) 0 to 0.8 ); the procedural mortality was $0.2 \%$ (two patients) including one patient who died following emergency bypass surgery. Two patients died from a cerebrovascular cause during their hospital admission. Coronary bypass surgery during the hospital stay was performed in eight patients $(0.8 \%, 95 \%$ CI 0.2 to -1.3$)$ : in five patients as an emergency procedure, in two on the same day as the angioplasty procedure, and in one on the next day. Repeat PTCA within 48 hours was performed in 11 patients $(1 \%, 95 \%$ CI 0.4 to 1.7). $Q$ wave myocardial infarction occurred in five patients, 22 patients had an enzymatically determined myocardial infarction. The procedure was unsuccessful but without complications in 47 patients $(4.6 \%, 95 \%$ CI 3.3 to 5.9$)$.

Table 2 Patient characteristics ( $n=1015$ patients)

\begin{tabular}{|c|c|c|}
\hline & $n$ & $\%$ \\
\hline Mean age (years) (SD) & $60(10.9)$ & \\
\hline Range & $25-84$ & \\
\hline Men & 745 & 73 \\
\hline Previous myocardial infarction & 516 & 51 \\
\hline Previous coronary angioplasty & 263 & 26 \\
\hline Previous coronary bypass surgery & 57 & 6 \\
\hline \multicolumn{3}{|l|}{ Risk factors } \\
\hline \multicolumn{3}{|l|}{$\begin{array}{l}\text { Family history of coronary artery } \\
\text { disease }\end{array}$} \\
\hline Hypercholesterolaemia & 570 & 56 \\
\hline Smoking & 422 & 42 \\
\hline Diabetes & 130 & 13 \\
\hline Hypertension & 418 & 41 \\
\hline Single vessel disease & 558 & 55 \\
\hline Multivessel disease & 457 & 45 \\
\hline Single lesion angioplasty & 628 & 70 \\
\hline Multilesion angioplasty & 270 & 30 \\
\hline Multivessel angioplasty & 117 & 12 \\
\hline \multicolumn{3}{|l|}{1510 Lesions (1.5/procedure) } \\
\hline \multicolumn{3}{|l|}{ Left anterior descending coronary } \\
\hline artery & 714 & 47 \\
\hline Right coronary artery & 442 & 29 \\
\hline Circumflex coronary artery & 329 & 22 \\
\hline Left main & 1 & 0.1 \\
\hline Saphenous vein graft & 24 & 2 \\
\hline De novo lesions & 1336 & 89 \\
\hline Restenotic lesions & 174 & 11 \\
\hline \multicolumn{3}{|l|}{ ACC/AHA classification } \\
\hline Type A & 40 & 3 \\
\hline Type B1 & 519 & 34 \\
\hline Type B2 & 601 & 40 \\
\hline Type C & 350 & 23 \\
\hline Eccentric & 1287 & 85 \\
\hline \multicolumn{3}{|l|}{ Proximal tortuosity: } \\
\hline Moderate & 629 & 42 \\
\hline Severe & 174 & 12 \\
\hline \multicolumn{3}{|l|}{ Angulation: } \\
\hline$>45^{\circ}$ & 416 & 28 \\
\hline$>60^{\circ}$ & 34 & 3 \\
\hline Calcification & 393 & 26 \\
\hline Thrombus & 68 & 5 \\
\hline Ostial location & 172 & 11 \\
\hline Side branch involvement & 430 & 29 \\
\hline Bifurcation lesion & 156 & 10 \\
\hline \multicolumn{3}{|l|}{ Coronary occlusion: } \\
\hline Total & 251 & 17 \\
\hline Functional & 95 & 6 \\
\hline Balloon angioplasty & 798 & 79 \\
\hline Stent implantation & $194^{\star}$ & 19 \\
\hline Directional atherectomy & $24^{\star}$ & 2 \\
\hline Rotational atherectomy & 3 & 0.3 \\
\hline
\end{tabular}

^Including four directional atherectomy procedures followed by stent implantation.

ACC/AHA, American College of Cardiology/American Heart Association. 
Table 3 Indications for prolonged observation $(n=87$ patients)

\begin{tabular}{lr}
\hline Severe dissection: failed or suboptimal stent & 42 \\
Occluded side branch & 20 \\
Angiographic thrombus/no reflow & 9 \\
Repeat PTCA < 4 hours observation & 5 \\
Perforation with guidewire & 3 \\
Suspected cerebrovascular accident & 3 \\
Other (operator's discretion) & 5 \\
\hline
\end{tabular}

Table 4 Multivariate preprocedural predictors of prolonged observation

\begin{tabular}{llll}
\hline & Odds ratio & $95 \% C I$ & p Value \\
\hline Ostial lesion & 1.6 & 1.2 to 2.5 & 0.0005 \\
PTCA > 1 lesion & 1.8 & 1.1 to 2.3 & 0.02 \\
Restenotic & 1.7 & 1.0 to 2.7 & 0.03
\end{tabular}

CI, confidence interval; PTCA, percutaneous transluminal coronary angioplasty.

\section{SHORT TERM OBSERVATION}

After a four hour observation period, 922 of 1015 patients $(90.8 \%, 95 \%$ CI 89.1 to 92.6$)$ were discharged and returned to the referring hospital. Among the patients allocated to short term observation, there were no deaths, and none of these patients had ECG or enzymatic evidence of myocardial infarction. No patients were returned to our centre for acute recurrent ischaemia before discharge from the referring hospital. All patients remained stable for at least three days.

PROLONGED OBSERVATION

Eighty seven patients were triaged to prolonged observation $(8.6 \%)$. The indications for prolonged observation are summarised in table 3 . Five patients were observed after emergency repeat angioplasty for out of laboratory closure during the four hour postprocedural observation period. The patients selected for prolonged observation were monitored, heparin treatment was continued in 80 of 87 patients, and the sheaths were left in place until the next day. The remaining seven patients were observed without heparinisation for various reasons such as wire perforation or a suspected cerebrovascular complication.

The group of patients selected for prolonged observation comprised two in-hospital deaths from a cerebrovascular accident, two patients who underwent coronary bypass surgery within 48 hours of angioplasty, and two with significant pericardial fluid after wire perforation, warranting drainage in one patient. Repeat coronary angioplasty during prolonged observation was performed as an emergency procedure in four patients, and after scheduled reangiography within 48 hours in three. Myocardial infarction, defined as creatine kinase $\mathrm{MB}$ elevation of more than twice the upper limit of

Table 5 Trends in the use of intracoronary stents during the study

\begin{tabular}{|c|c|c|c|c|}
\hline & \multicolumn{2}{|l|}{ Period I } & \multicolumn{2}{|l|}{ Period II } \\
\hline & All & Observed (\%) & All & Observed (\%) \\
\hline Number of patients & 505 & $47(9.3)$ & 504 & $40(7.9)$ \\
\hline Stents $(\%)$ & $57(11.2)$ & $19(33.3)$ & $130(25.8)$ & $22(16.9)$ \\
\hline Elective & 30 & $4(13.3)$ & 58 & $4(6.9)$ \\
\hline Unsatisfactory result & 15 & $6(40.0)$ & 48 & $8(16.6)$ \\
\hline "Bail out" & 12 & $9(75.0)$ & 24 & $10(41.6)$ \\
\hline
\end{tabular}

normal and/or development of new $\mathrm{Q}$ waves, was encountered in 27 patients (creatine kinase $\mathrm{MB}>2$ but $<3$ times the upper limit in six; $>3$ times in 21 ; with new $\mathrm{Q}$ waves in five). The postprocedural course was uneventful in 47 of the 87 patients selected for prolonged observation $(54 \%)$.

PREDICTORS OF PROLONGED OBSERVATION

On univariate analysis, triage to prolonged observation was associated with the following preprocedural variables: previous PTCA, multilesion and multivessel angioplasty, angioplasty of restenotic lesion; and with the following angiographic variables: ACC/AHA lesion type $\mathrm{B} 2$ and $\mathrm{C}$, ostial lesion location, and presence of calcification. None of the other preprocedural clinical or lesion specific variables was significantly associated with prolonged observation. On multivariate analysis, ostial lesion location, PTCA of more than one coronary lesion, and PTCA of a restenotic lesion were identified as independent preprocedural predictors of prolonged observation (table 4). Statistical analysis was performed per patient as well as per lesion, to avoid possible diluting effects of successfully dilated lesions in patients who underwent multiple lesion angioplasty and were observed for a complication related to one lesion. These two approaches did not have significantly different associations. Prolonged observation was more likely to be needed after stent implantation, but this was related to "procedural" stenting for an unsatisfactory angioplasty result or its use as a bail out device. No such association was documented for elective stent implantation. To take into account the increased use of stents, the effect of stent implantation on the need for prolonged observation was evaluated separately in the first and the second half of the study cohort. Table 5 shows the overall increase in the use of stents in the second half of the study, and the decrease in the number of patients allocated to prolonged observation after stent implantation for unsatisfactory angioplasty or as a bail out device.

PREDICTORS OF COMPLICATIONS

Forty of 87 patients triaged to prolonged observation developed a complication, compared with none of the 922 patients triaged to early discharge. After univariate analysis, the following preprocedural and procedural variables were entered in a multivariate logistic regression analysis to identify independent predictors of complications: sex, diabetes, vessel selected for angioplasty, multilesion angioplasty, restenotic lesion, ostial location, calcification, bifurcation lesion, lesion severity, lesion length $>10 \mathrm{~mm}$, acute/temporary occlusion, side branch occlusion, thrombus, embolisation, local versus extensive dissection, dissections classified according to the NHLBI, any non-elective stent, bail out stent, and stenting for an unsatisfactory result. Multivariate logistic regression identified six variables as independent predictors of a complicated clinical course (table 6).

The patients were ranked according to the score of the regression model and divided into 
Table 6 Multivariate predictors of complications

\begin{tabular}{lllll}
\hline Predictor & Odds ratio & $95 \%$ CI & p Value & Risk points \\
\hline Acute (temporary) closure & 9.7 & 4.4 to 21.4 & 0.0001 & 4 \\
Side branch occlusion & 8.9 & 3.4 to 23.7 & 0.0001 & 4 \\
No angiographic success & 5.1 & 2.4 to 11.0 & 0.0002 & 3 \\
Female sex & 3.1 & 1.7 to 5.7 & 0.0001 & 2 \\
Any unplanned stent & 2.8 & 1.4 to 5.9 & 0.019 & 2 \\
Ostial lesion & 2.2 & 1.0 to 4.7 & 0.034 & 2
\end{tabular}

${ }^{\star}$ Risk points for a simplified risk function; the weights are assigned in proportion to the logarithm of the corresponding odds ratio from the multivariate analysis. The patients' total risk score is calculated as the sum of the risk points of all properties that apply.

CI, confidence interval.

Table 7 Patients, prolonged observation, and complications by total risk score

\begin{tabular}{lllll}
\hline Risk & $\begin{array}{l}\text { Sum of } \\
\text { risk points }\end{array}$ & $\begin{array}{l}\text { Number of } \\
\text { patients }\end{array}$ & $\begin{array}{l}\text { Observed } \\
\text { patients (\%) }\end{array}$ & $\begin{array}{l}\text { Complicated } \\
\text { patients }\end{array}$ \\
\hline Very low & 0 & 475 & $12(2.5)$ & 2 \\
Low & 2 & 296 & $20(6.8)$ & 7 \\
Medium & $3-4$ & 146 & $18(12.3)$ & 5 \\
High & $5-6$ & 63 & $19(30.1)$ & 11 \\
Very high & $>6$ & 29 & $18(62.0)$ & 15 \\
\hline
\end{tabular}

strata of very low, low, medium, high, and very high risk, corresponding to $0,2,3-4,5-6$, and more than 6 risk points. Table 7 shows the distribution of the patients over the risk categories. Almost half the patients (475) had no risk points. Twelve of these patients were triaged to prolonged observation and two developed a complication. Both were patients who underwent emergency repeat PTCA within four hours after the procedure for recurrent ischaemia, resulting in creatine kinase $\mathrm{MB}$ elevations. Of 20 observed patients in the low risk group, seven had complications including a fatal cerebrovascular accident, emergency repeat PTCA for acute vessel closure after allocation for prolonged observation, side branch occlusion, and embolisation with subsequent creatine kinase $\mathrm{MB}$ elevations. The majority of patients at very high risk (18 of 29) were kept for prolonged observation. All but three developed a complication. The patients discharged after four hours with a very high risk score (11 of 29) included two patients with unsuccessful angioplasty for a chronic total occlusion and nine with a good result after bail out stent implantation for temporary vessel closure. Complications occurred in all risk groups, although with a lower incidence rate in the lower risk categories.

\section{Discussion}

Our study shows that a four hour observation period is safe after elective coronary angioplasty. The immediate procedural results allow effective triage of patients to short term or prolonged observation in order to anticipate postprocedural complications effectively. In all, $90.8 \%$ of the included patients were discharged after four hours of observation and had an uncomplicated course. The observation period was prolonged in five patients following emergency repeat coronary angioplasty for acute recurrent ischaemia within four hours of the procedure, indicating that this period is apparently sufficient for short term observation. In a total of 87 patients $(8.5 \%)$, prolonged observation was considered necessary, and complications were encountered in 40 of these.
Risk factors for coronary angioplasty related complications have been assessed in several retrospective and prospective reports. ${ }^{4-9}$ However, the vast majority of patients undergoing angioplasty nowadays would have been excluded from short term observation because of the risk estimates derived from those studies. Furthermore, a wide variation in the estimated risk has been reported, precluding accurate risk stratification in the individual patient..$^{101120}$ In the present study, the opposite approach was chosen. Patients scheduled for elective coronary angioplasty were prospectively included for short term observation, independently of the clinical characteristics or angiographic features formerly associated with a higher risk of complications.

PREDICTORS OF PROLONGED OBSERVATION

Prolonged observation, at the discretion of the operator, was associated by univariate analysis with various previously reported preprocedural risk factors for adverse outcome, such as multilesion angioplasty, ${ }^{4-6} \mathrm{ACC} / \mathrm{AHA}$ lesion type B2 and C generally, ${ }^{491820}$ ostial lesion location, ${ }^{5618}$ and the presence of lesion calcification. ${ }^{10}$ Other previously reported predictors of an adverse outcome, such as longer lesions, ${ }^{5}{ }^{1021}$ lesion severity, ${ }^{810}$ and right coronary artery location, ${ }^{10}$ were not related to triage for prolonged observation. The only independent predictors of prolonged observation identified by multivariate analysis were ostial lesion location, multilesion angioplasty, and angioplasty of a restenotic lesion. However, the calculated odds ratios show that these factors are relatively weak predictors of prolonged observation.

Previous PTCA appeared to be related to prolonged observation. This was partly accounted for by angioplasty of restenotic lesions, which was highly associated with prolonged observation in both univariate and multivariate analysis. The explanation for this phenomenon remains speculative: the choice of different devices for restenotic lesions did not sufficiently explain the observed association, as neither atherectomy nor elective stent implantation was associated with prolonged observation by univariate analysis. Although patients undergoing angioplasty for restenosis were more often selected for prolonged observation than patients with de novo lesions, angioplasty of a restenotic lesion was not associated with a higher complication rate. This corresponds with previous reports indicating that angioplasty of restenotic lesions bears a similar or even reduced risk compared to de novo lesions. $^{22}$

Elective stent implantation was not associated with prolonged observation, in contrast to unplanned stenting for an unsatisfactory result of angioplasty or as a bail out device. The effect of stent implantation on the need for prolonged observation was evaluated separately in the first and the second half of the study, because we anticipated changes in the operators' acceptance of stenting for short term observation with the increased use of stents (table 5). This evaluation showed the evolving role of elective 
stent implantation followed by short term observation. Furthermore, there was a trend towards an increase in short term observation after "unplanned" stenting, indicating the growing confidence of the operators in the adequacy of coronary stenting for bail out indications.

PREDICTORS OF COMPLICATIONS

Postprocedural complications included in the analysis were death, the need to perform bypass surgery or repeat coronary angioplasty in the days following the procedure, myocardial infarction, and pericardial drainage for perforation. The strongest predictors of such postprocedural complications were temporary vessel closure, side branch occlusion, and an unsuccessful angiographic angioplasty result - that is, procedural variables. The only preprocedural clinical and lesion morphology variables significantly related to postprocedural complications by multivariate analysis were female sex and ostial lesion location. Again, these two variables were weaker predictors of complications than the procedure related factors. This emphasises the limited value of preprocedural clinical and angiographic characteristics in the exclusion of patients from a short term observation angioplasty protocol. Selection for prolonged observation to anticipate subsequent complications can be performed on the immediate postprocedural findings, irrespective of "high risk" clinical or morphological characteristics.

Our analysis shows that we were able to select those patients who were at risk of complications. Table 7 indicates that it would be difficult to improve our triage results by reducing the number of patients who were observed but did not actually have subsequent complications that required a prolonged hospital stay. If prolonged observation was withheld in patients with a very low or low risk, another 12 or 20 patients would have been discharged at the expense of two or seven serious complications, respectively, after discharge from the intervention centre.

\section{STUDY LIMITATIONS}

This study was a single centre evaluation and the results may not be transferable to centres with a different patient population for elective coronary angioplasty. Nevertheless, the main clinical and angiographic characteristics of the study patients are in accordance with those of patients reported in most angioplasty registries. $^{268}$

Our study protocol did not allow us to draw any conclusions about the appropriateness of continuing heparin treatment in all patients selected for prolonged observation. Moreover, the anticoagulation treatment did not include the administration of IIb/IIIa receptor antagonists, which are potentially useful in this class of patient. ${ }^{23} 24$

It is conceivable that many clinical and angiographic characteristics previously labelled "adverse" could not be related to prolonged observation or postprocedural complications because the sample size was too small. The inability to achieve statistical significance has been emphasised by others in studies evaluating morphological lesion characteristics of acute closure and angioplasty complications. ${ }^{10}{ }^{11}$ However, our study was not primarily designed to identify predictors of angioplasty related complications. The aim of the study was to evaluate the effectiveness of the triage to short term $v$ prolonged observation and to identify preprocedural and procedural variables that would allow appropriate selection of patients for prolonged observation, in order to anticipate complications.

\section{CLINICAL IMPLICATIONS}

Our study shows that a four hour observation period is safe after elective coronary angioplasty. The immediate procedural result allows effective triage of patients for short term or prolonged observation anticipating postprocedural complications, irrespective of "adverse" clinical or morphological characteristics. In a previous report, we demonstrated the safety of the use of 5000 IU of heparin in elective coronary angioplasty without an increased risk of ischaemic complications. ${ }^{25}$ Similar results with a standard dose of 5000 IU of heparin have been reported by others. ${ }^{26}{ }^{27}$ This heparin dose enables immediate sheath removal, which may be particularly useful in a patient population which appears eligible for short term observation. Triage of patients as performed in the present study, in conjunction with a low dose heparin anticoagulation regimen, may facilitate early discharge and outpatient angioplasty in the majority $(90 \%)$ of patients scheduled for elective coronary angioplasty.

We gratefully acknowledge the technical and nursing staff of our Cardiac Catheterisation Laboratory (chief, M G H Meesterman, RN) for skilled assistance.

1 Ellis SG, Miller DP, Brown KJ, et al. In-hospital costs of percutaneous coronary revascularisation. Circulation percutaneous

2 Detre KM, Holmes DR, Holubkow $\mathrm{R}$, et al, and the Co-investigators of the National Heart, Lung, and Blood Institute's Percutaneous Transluminal Coronary Angioplasty Registry. Incidence and consequences of periprocedural occlusion of the 1985-1986 National Heart, Lung and Blood Institute's Percutaneous Transluminal Coronary Angioplasty Registry. Circulation 1990;82:739-50.

3 Steffino G, Meier B, Finci L, et al. Acute complications of elective coronary angioplasty: a review of 500 consecutive procedures. Br Heart $\mathcal{F}$ 1988;59:151-8.

4 De Feyter PJ, Van den Brand M, Laarman GJ, et al. Acute coronary artery occlusion during and after percutaneous,
transluminal coronary angioplasty. Frequency, prediction, management and follow-up. Circulation 1991;83:927-36.

5 Ellis SG, Roubin GS, King SB, et al. Angiographic and clinical predictors of acute closure after native vessel coronary angioplasty. Circulation 1988;77;372-9.

6 Ellis GE, Vandormael MG, Cowley MJ, et al, and the Multivessel Angioplasty Prognosis Study Group. Coronary morphologic and clinical determinants of procedural outcome with angioplasty for multivessel coronary disease: implications for patients selection. Circulation 1990;82: implications

7 Savage MP, Goldberg S, Hirschfeld JW, et al. Clinical and angiographic determinants of primary coronary angioplasty success. F Am Coll Cardiol 1991;17:22-8.

8 Myler RK, Shaw RE, Stertzer SH, et al. Lesion morphology and coronary angioplasty; Current experience and analysis. 7 Am Coll Cardiol 1992;19:1641-52.

9 Lincoff AM, Popma JJ, Ellis SG, et al. Abrupt vessel closure complicating coronary angioplasty: clinical, angiographic

and therapeutic profile. A Am Coll Cardiol 1992;19:926-35. of abrupt vessel closure after angioplasty in an individual patient. F Am Coll Cardiol 1994;24:1004-11.

11 Tan K, Sulke N, Taub N, et al. Clinical and lesion morphologic determinants of coronary angioplasty success and
complications: current experience. $\mathcal{F}$ Am Coll Cardiol 1995; 25:855-65. 
12 Black AJR, Namay DL, Niederman AL, et al. Tear or dissection after coronary angioplasty: morphologic correlates of an ischemic complication. Circulation 1989;79:1035-42.

13 Huber MS, Mooney JF, Madison J, et al. Use of a morphologic classification to predict clinical outcome after dissection from coronary angioplasty. Am f Cardiol 1991;68:467-71.

14 Agrawal SK, Ho DSW, Liu MW, et al. Predictors of thrombotic complications after placement of the flexible coil stent. Am f Cardiol 1994;73:1216-19.

15 Braunwald E. Unstable angina, a classification. Circulation 1989;80:4104.

16 Principal investigators of CASS and their associates. The National Heart, Blood and Lung Institute Coronary Artery Surgery Study. Circulation 1981;63:131-9.

17 TIMI Study Group. The Thrombolysis in Myocardial Infarction trial. N Eng f Med 1985;313:342-7.

18 Ryan TJ, Faxon DP, Gunnar RP, and ACC/AHA Task Force. Guidelines for percutaneous transluminal angioporce. G Am Coll Cardiol 1988;12:529-45.

19 Dorros G, Cowley MJ, Simpson J, et al. Percutaneous transluminal coronary angioplasty: report of complications from The National Heart, Blood and L
istry. Circulation 1983;67:723-30.

20 Budde T, Haude M, Hopp HW, et al. A prognostic computer model to predict individual outcome in interventional cardiology; The Intervent Project. Eur Heart $\mathcal{F}$ 1997;18 1611-19.
21 Topol EJ, Ellis SG, Fishman J, et al. Multicenter study of percutaneous transluminal coronary angioplasty for of percutaneous transluminal coronary angioplasty for right coro.

22 Dimas AP, Grigera F, Arora RR, et al. Repeat coronary angioplasty as treatment for restenosis. $\mathcal{F} \mathrm{Am}$ Coll Cardiol 1992;19:1310-14

23 Lincoff AM, Cheng JE, Califf RM, et al, for the PROLOG investigators. Standard versus low dose weight-adjusted heparin in patients treated with the platelet glycoprotein IIb/IIIa receptor antibody fragment abciximab (c7E3 Fab) during percutaneous coronary revascularisation. Am f Cardiol 1997;79:286-91.

24 The Epilog Investigators. Platelet glycoprotein IIb/IIIa receptor blockade and low-dose heparin during percutaneous coronary revascularisation. N Eng f Med 1997:336: 1689-96.

25 Koch KT, Piek JJ, de Winter RJ, et al. Safety of low-dose heparin in elective coronary angioplasty. Heart 1997; heparin in

26 Vainer J, Fleisch M, Gunnes P, et al. Low-dose heparin for routine coronary angioplasty and stenting. Am $\mathcal{F}$ Cardiol

27 Kiemeneij F, Laarman GJ, Odekerken D, et al. A randomized comparison of transluminal coronary angioplasty by the radial, brachial and femoral approaches: the access study. F Am Coll Cardiol 1997;29:1269-75.

\section{Potentially fatal atrial pacemaker lead disruption detected by fluoroscopic surveillance}

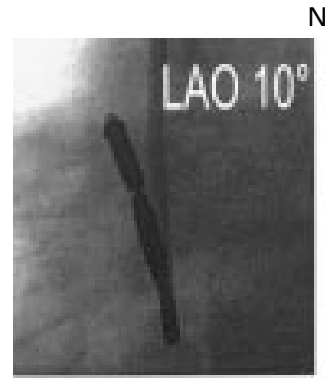

November 1998
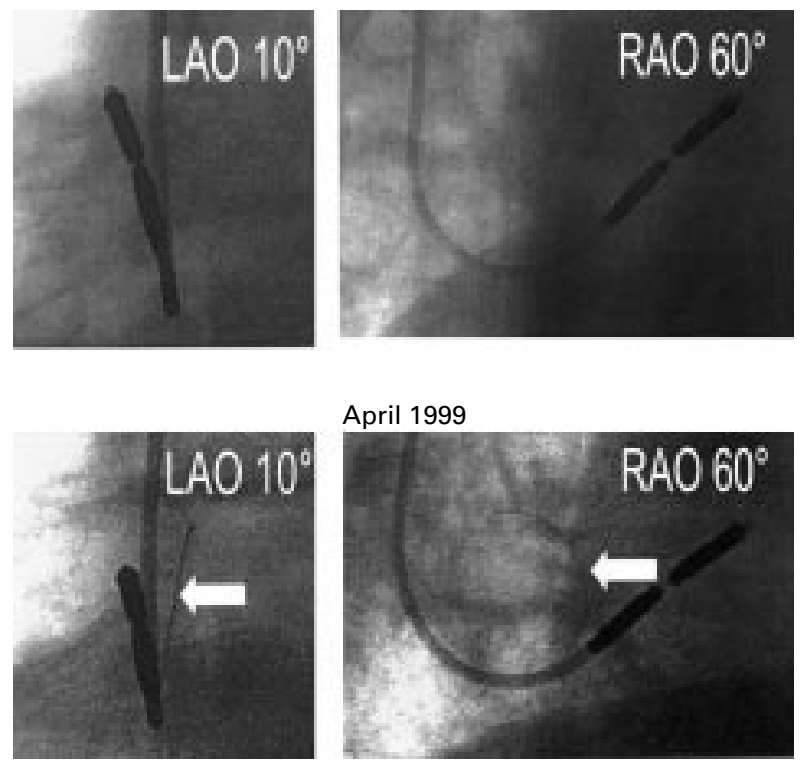

April 1999

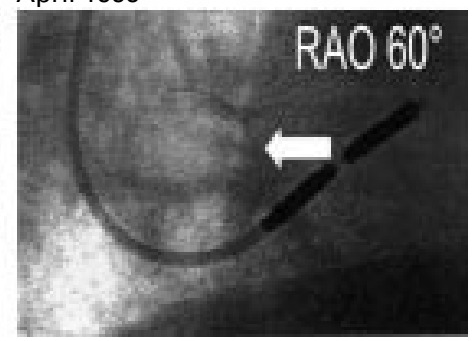

In March 1994, a 66 year old man underwent atrial pacemaker implantation using an Accufix 330-801 lead (Telectronics) for sinus arrest. After the manufacturer's recall, the atrial lead was checked by fluoroscopy every six months as recommended. In April 1999, five years after implant, protrusion of the J-shaped retention wire through the lead

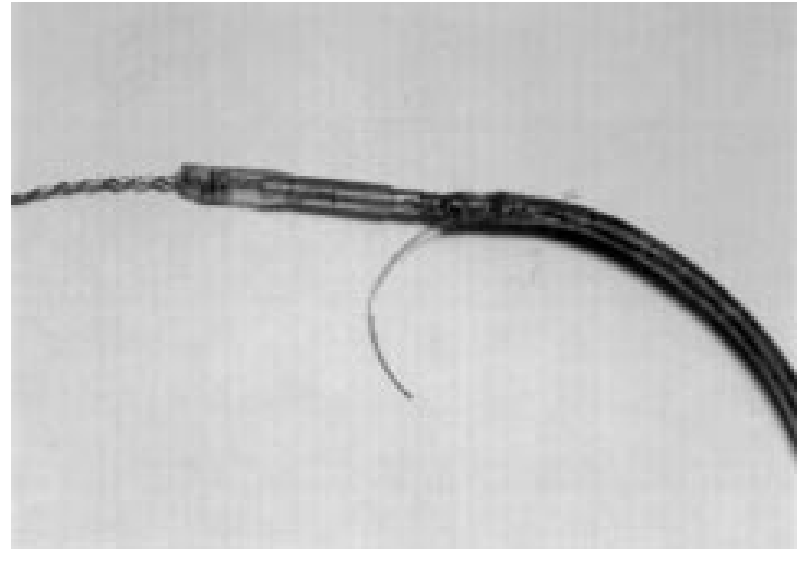

insulation into the right atrium was detected (left). This complication is associated with a significant risk of cardiac perforation, tamponade, and sudden death. The lead and the $\mathrm{J}$ retention wire were therefore extracted transvenously with some unraveling of the conductor, but without complication (above). A replacement pacing system was implanted. Potentially life threatening disruption of an Accufix lead late after implant may initially be asymptomatic and not associated with pacemaker malfunction. Surveillance fluoroscopy may be life saving.

DIANA A GOROG DAVID C LEFROY 